\title{
Determinar Estrategias Para La Administración De Riesgos Laborales Y Su Impacto En La Competitividad De Las Empresas
}

\author{
Carlos Alfredo Juárez Cerros, Ingeniero Industrial \\ Francisco Arturo Bribiescas Silva, PhD \\ Universidad Autónoma de Ciudad Juárez, México
}

doi: 10.19044/esj.2016.v12n31p81 URL:http://dx.doi.org/10.19044/esj.2016.v12n31p81

\begin{abstract}
To be a competitive company, it's necessary that the quality has a high sense of importance for managers; some indicators such as market impact, the gradual increase in the interest of the academic community, the changes in its principles and practices and the organization of experts in the field, show the progress of this topic in organizations.

Companies seek management systems that can be easily implemented, and in turn manage them effectively according to their different activities to achieve remain in an increasingly competitive environment; the key to effectively manage quality and risk prevention, is how to meet regulatory requirements and increasingly demanding needs of its main audiences: customers, society and workers, and at the same time allow them to be competitive.

In this paper some authors suggest a number of tools for managing occupational hazards that can be used in any business, in order to identify different analysis methodologies and risk controls, based on them are consulted, develop a process that contributes to the continuous improvement of working environments for workers; it is also necessary to implement surveys in the manufacturing sector to corroborate the impact of occupational risk management in the competitiveness of enterprises.
\end{abstract}

Keywords: Strategy, Labor risks, Competitiveness, Quality

\section{Resumen}

Para tener una empresa competitiva, es necesario que la calidad tenga un alto sentido de importancia para los directivos; algunos indicadores como el impacto en el mercado, el progresivo aumento del interés de la comunidad académica, los cambios sufridos en sus principios y prácticas, y la organización de los expertos en la materia, muestran el avance de este tópico en las organizaciones. 
Las empresas, buscan sistemas de gestión que se puedan implantar de manera sencilla, y a su vez, administrarlos de forma eficaz acorde a sus diferentes actividades para lograr permanecer en un entorno cada vez más competitivo; una clave para gestionar eficazmente la calidad y la prevención de riesgos laborales, es saber cómo satisfacer los requisitos reglamentarios y las demandas cada vez más exigentes de sus principales destinatarios: los clientes, la sociedad y los trabajadores, y que al mismo tiempo les permita ser competitivas.

En este documento, se consultan algunos autores que plantean una serie de herramientas para la administración de riesgos laborales y que pueden ser utilizadas en cualquier tipo de negocio, con el objetivo identificar diferentes metodologías de análisis y control de riesgos y, con base en ellas, desarrollar un proceso que aporte al mejoramiento continuo de espacios laborales para los trabajadores; asimismo, es necesario aplicar encuestas en el sector de la industria manufacturera para corroborar el impacto que tiene la administración de riesgos laborales en la competitividad de las empresas.

Palabras clave: Estrategias, Riesgos laborales, Competitividad, Calidad

\section{Introducción}

En años anteriores a la Revolución Industrial, el trabajo en las fábricas era escaso, los productos eran fabricados artesanalmente y en lugares que eran propiedad de los mismos artesanos; dicha revolución se desarrolló en Inglaterra a partir del siglo XVII, y como consecuencia de ella se incrementó el trabajo en las fábricas (Garachana, 1999, pp. 17-18) en condiciones mínimas de salud y seguridad, en parte debido a la cantidad, pero principalmente por la carencia de una cultura de seguridad eficiente, tanto de parte de los trabajadores como de los empleadores. Algunos autores como Gallegos (2012, p. 46) indican que las dos terceras partes de los obreros eran mujeres y niños explotados, y muchos de ellos sufrían lesiones, mutilaciones o bien morían en accidentes trágicos, pero recurrentes.

Con el incremento de los accidentes y las enfermedades laborales, fue necesario elaborar estudios e implementar estrategias de seguridad industrial; en Inglaterra, entre 1833 y 1867, se dieron a conocer las primeras leyes que ayudarían a mejorar las condiciones laborales, y en Alemania, en 1869 se creó el primer Seguro Social para los trabajadores (Cavassa, 1986, pp. 2425). En México, en 1911 se creó el Departamento del Trabajo, pero fue necesario esperar 20 años para que la primera Ley Federal del Trabajo se promulgara [1931]. Posteriormente, debido a la complejidad del sector laboral, en 1940 el Presidente Manuel Ávila Camacho promulgó una nueva Ley de Secretarías de Estado, en la que se estableció que el Departamento del Trabajo se convertía en la Secretaría del Trabajo y Previsión Social 
[STPS], cuya estructura y organización permitió responder a las demandas sociales (Secretaría del Trabajo y Previsión Social, 2015).

Con lo anterior, es posible afirmar que a medida que creció la industria manufacturera, los controles y las regulaciones gubernamentales y no gubernamentales para la integración de modelos de administración de riesgos laborales a los estándares internacionales, evolucionaron con el fin de proporcionar condiciones laborales más dignas para los trabajadores.

\section{Planteamiento del problema}

Una buena administración de riesgos se centra en la identificación y el tratamiento de esos eventos, con el propósito de incrementar la probabilidad de éxito y reducir la de fracaso y la incertidumbre de lograr los objetivos y metas generales de la organización (Cuello, Pallares, \& Wehdeking, 2008, p. 97). Así, la seguridad emerge como una necesidad social para prevenir daños personales causados por las condiciones laborales; además, existen otro tipo de motivadores para gestionar la administración de riesgos, entre los que se encuentran factores asociados con aspectos humanos, legales y económicos (Cortés, 2007, pp. 105-106), los cuales se muestran en la Figura 1.

Figura 1. Motivaciones para la administración de riesgos.

Humanas
- Para el accidentado, el haber
sufrido un accidente de
trabajo o una enfermedad
laboral repercute en su salud
física y psicológica, el
empleado puede disminuir su
capacidad productiva a causa
de una lesión que restrinja su
libertad de movimiento, o por
temor a sufrir nuevamente un
accidente.
-Para la familia de la víctima,
además del dolor físico y
solidario con el accidentado,
repercute en consecuencias
económicas que se derivan del
daño. Sin mencionar que la
consecuencia del accidente
haya terminado en una
fatalidad y signifique la
pérdida de un miembro de la
familia.
- Para el ambiente laboral,
puede verse afectado el
aspecto emocional de los
compañeros laborales.
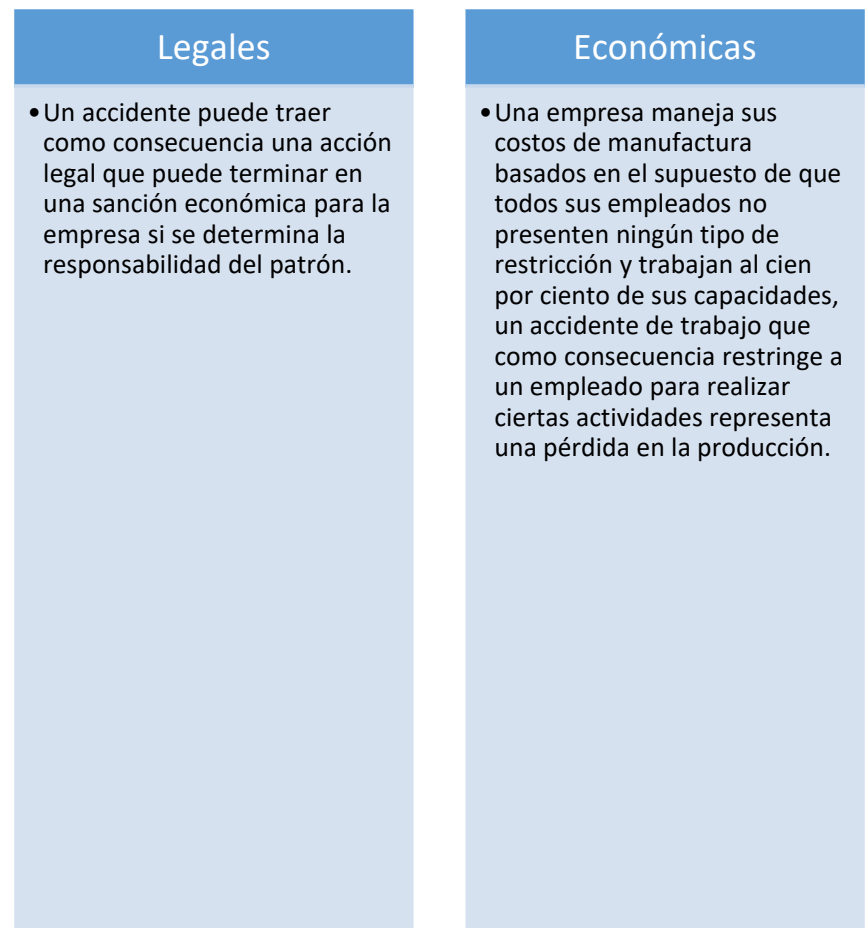

Fuente: Elaboración propia con base en Cortés (2007, pp. 105-106). 


\section{Revisión de la literatura: El Checklist Manifesto}

Gawande (2010, pp. 8-10) en su libro The Checklist Manifesto, presenta una serie de hechos en los que el uso de las listas de control llamadas checklist, ha resuelto de manera exitosa situaciones que pudieron desencadenar en accidentes graves o pérdida de vidas, principalmente en el ámbito de la medicina y la aviación; como en el caso de un avión Boeing 299 de cuatro motores que en 1935 la Fuerza Aérea de Estados Unidos no permitió su despegue debido a que no paso las inspecciones iniciales, salvando la vida del piloto; otro caso fue un estudio de investigación de tres meses en un hospital de Michigan, la cual revelo que se redujeron un 66\% las tasas de infecciones desde que se empezaron a utilizar estas listas de verificación por el personal del hospital; asimismo, se ha atribuido el crédito a la utilización de los checklist en otras áreas como la construcción y la ingeniería, en donde se ha disminuido la tasa de errores.

\section{Análisis sobre la gestión integrada de la calidad, el medio ambiente y la seguridad en los proyectos industriales}

Este estudio cualitativo realizado por Saenz-Calcedo et al. (2015, pp. 141-143), busca la posibilidad de generar un sistema integrado de calidad, medio ambiente y seguridad, utilizando los estándares internacionales ISO 9001:2015 para calidad, ISO 14001:2015 para medio ambiente y OHSAS 18001:2007 para salud y seguridad en el trabajo, con el objetivo de tener beneficios económicos sustentables desde la perspectiva de responsabilidad social del negocio; asimismo, este es implementado con la ayuda del ciclo de Deming para la mejora continua [Figura 1]; la calidad, el medio ambiente y la seguridad han tenido un desarrollo paralelo en la industria, mientras que la seguridad depende de los recursos humanos, la calidad hace que las operaciones y el medio ambiente se encuentren en áreas técnicas.

Figura 2. Integración de la calidad, el medio ambiente y la seguridad.

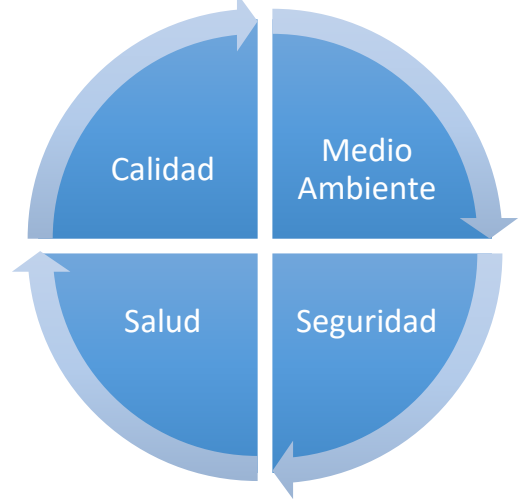

Fuente: Elaboración propia con base en Saenz-Calcedo et al. (2015, p. 141). 
La calidad se ha desarrollado con fuerza impulsada por la competencia, por la necesidad de mejorar la competitividad de las empresas, mientras que el establecimiento de regulaciones y la presión de los sindicatos del gobierno han aumentado la seguridad, mientras que el medio ambiente se ha hecho por la ley y la sociedad, por lo que un sistema integrado garantiza que cualquier empresa puede gestionar sus actividades y procesos industriales, de una forma sostenible y respetuosa del medio ambiente con la calidad del medio ambiente (Saenz-Calcedo, González, \& Et al, 2015, p. 141).

\section{Habilidades de prevención de errores para personal de primera línea de General Eletric}

En un manual elaborado para la empresa General Electric, Tony Muschara (2010, p. 8) hace énfasis en que los trabajadores deben tener precaución en sus lugares de trabajo, tomando en cuenta que "Nada es siempre como parece”. Los encargados de la seguridad industrial deben estar siempre conscientes de la presencia de amenazas ocultas en el entorno de trabajo, ya que la mayoría de los eventos ocurren durante las llamadas actividades "rutinarias"; por lo general, las personas piensan erróneamente que "simple” o "rutinario" significa que no hay riesgos, sin embargo, los especialistas siempre se preocupan por posibles fallas, y toman una serie de pasos para administrar el riesgo del error, el cual se enfoca principalmente en los riesgos de su trabajo, y que normalmente incluye las siguientes practicas:

1. Anticipar lo que puede ir mal - saber qué esperar.

2. Monitorear las actividades o procesos que tengan riesgos importantes - saber a qué poner atención.

3. Responder con los medios adecuados para evitar fallas y hacer frente a las consecuencias del error - saber qué hacer.

4. Aprender para mejorar la resiliencia del sistema a un error humano saber qué cambiar.

\section{Técnicas de prevención de riesgos laborales}

Para Menéndez (2007, pp. 75-78), la seguridad del trabajo presenta formas de actuación distintas y definidas para detectar y corregir los riesgos de trabajo, las cuales pueden ser denominadas como técnicas de seguridad, y son apoyadas en los factores causantes de accidentes previos se recurre a una serie de técnicas para prevenirlos que generalmente tienen dos finalidades diferentes: suprimir el riesgo y proteger al operario.

Dentro de las técnicas se pueden encontrar dos grupos bien definidos: las técnicas analíticas y las técnicas operativas, de las cuales, las analíticas pueden ser clasificadas como previas, o posteriores al accidentes; las técnicas previas consisten en tomar medidas antes de que ocurra un accidente, 
llevando a cabo inspecciones de seguridad, análisis de trabajo, análisis estadísticos, entre otros; mientras que las técnicas posteriores al accidente incluyen una investigación y registro del suceso, y la notificación mediante boletines a los empleados.

Asimismo, las técnicas operativas inciden en los factores técnicos y los humanos, los primeros van desde la concepción [planificación industrial y diseño de equipos], hasta los de corrección [sistemas de seguridad, protección personal, señalización, mantenimiento preventivo, entre otros]; mientras que los factores humanos abarcan desde la selección de personal, hasta los cambios en el comportamiento (Menéndez, 2007, pp. 75-78).

\section{Del dominó al queso suizo. La evolución del campo de la seguridad laboral}

La propuesta que presentan Conesa, García y Lamata (2012, pp. 101102) en este artículo, es un modelo que muestra una variedad de elementos que intervienen, así como su dependencia, para que un accidente no suceda; asimismo, permite tener una visión de la situación en tiempo real de seguridad de una empresa, facilitando la toma de decisiones en la planeación de los esfuerzos para la solución de problemas.

La teoría de Reason o del “Queso Suizo” muestra rebanadas de queso como las capas de protección hacia un potencial accidente laboral; sus agujeros representan los fallos humanos o del sistema que permiten que el incidente se abra paso para convertirse en un accidente; cabe mencionar que los huecos no son estáticos, sino que se mueven sobre la rebanada en la que se encuentran (Conesa, García, \& Lamata, 2012, pp. 101-102).

\section{La pirámide de accidentabilidad de Frank E. Bird}

Frank E. Bird, Jr., se interesó en un estudio realizado por H. W. Heinrich en su libro Industrial Accident Prevention de 1931, donde se muestra la relación; una lesión mayor a 29 lesiones menores a 300 nolesiones [1-29-300], por lo cual, Bird decidió realizar un estudio similar en 1969, en el cual, se analizaron 1'753,498 accidentes reportados por 297 compañías de diferentes ramas industriales y con un aproximado de 1'750,000 empleados quienes trabajaron más de tres mil millones de horas; el estudio reveló las siguientes relaciones de los accidentes reportados: por cada lesión mayor [resultando en una fatalidad, incapacidad del empleado, tiempo perdido o tratamiento médico] hubo 9.8 reportes de lesiones menores [requiriendo solo primeros auxilios]; el 20\% de las compañías indicaron que se investigaron todos los accidentes y el $80 \%$ mencionaron que investigaron todos los accidentes mayores; el análisis final indicó que 30.2 accidentes fueron reportados por cada accidente mayor; parte del estudio involucró cientos de horas de entrevistas confidenciales con los supervisores sobre la 
ocurrencia de los accidentes bajo diferentes circunstancias que pudieron terminar en una lesión (Radvanska, 2010, p. 74); finalmente, el análisis de estas entrevistas indicaron una relación de aproximadamente 600 incidentes por cada lesión grave; la relación 1-10-30-600 puede ilustrarse en forma de una pirámide que representa los accidentes y los incidentes con los entrevistadores, y no el número total de accidentes o incidentes que se han producido realmente, como se muestra en la Figura 3.

Figura 3. Pirámide de accidentabilidad de Frank E. Bird.

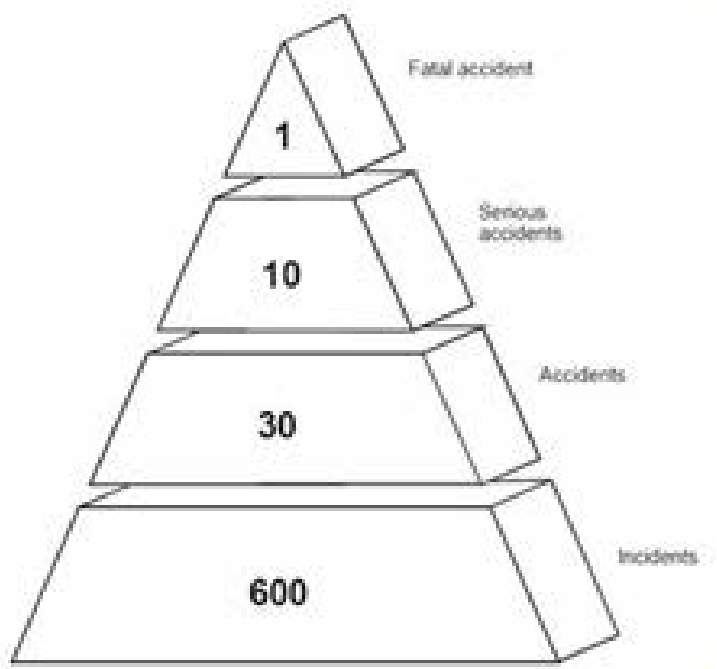

Fuente: Professional Safety (2007, p. 29).

Como punto importante, cabe destacar que las lesiones mayores son eventos poco frecuentes y combatiendo los eventos menos graves se previene que ocurran los serios. Los líderes de seguridad han hecho hincapié en que estas acciones son más efectivas cuando son dirigidas a incidentes menores con alto potencial de pérdida (Radvanska, 2010, p. 29).

\section{Mapas de riesgos y sus metodologías}

En su libro "Técnicas de prevención laborales: seguridad e higiene en el trabajo”, Tema 31, Cortés (2007, págs. 539-558) propone un documento que contiene información sobre los riesgos laborales existentes en una empresa llamado "mapa de riesgos", el cual permite identificar, localizar y valorar los peligros existentes, así como conocer el grado de exposición a los que están sometidos los trabajadores; de acuerdo con esto, se puede definir sus objetivos, los cuales se reducen a:

a) Identificar, localizar y valorar los riesgos existentes en una determinada empresa y las condiciones de trabajo relacionadas con ellos.

b) Conocer el número de trabajadores expuestos a los diferentes riesgos en función de departamentos o secciones, horarios y turnos. 
Para la elaboración del mapa de riesgos habrá de diseñarse previamente una serie de líneas de investigación y estudios que tiendan a conocer todos los datos y que permitan detectar los riesgos inherentes a los mismos, por lo cual, el autor propone una serie de aspectos a considerar para su realización, los cuales pueden ser agrupados conforme la situación a evaluar, como lo son las condiciones de seguridad, condiciones de higiene del trabajo, medio ambiente de trabajo, cargas físicas y mentales, aspectos psicosociales, entre otros; asimismo, para realizar este trabajo es necesario disponer de una serie de encuestas que, de forma simple y concreta, recaben información de los diferentes departamentos o secciones, así como de los tiempos de exposición.

\section{Programa de Autogestión en Seguridad y Salud en el Trabajo}

Con el objetivo de promover que las empresas en México instauren y operen un Sistema de Administración en Seguridad y Salud en el Trabajo, con base a estándares nacionales e internacionales y con el fin de disminuir los accidentes y enfermedades de trabajo, la Secretaría de Trabajo y Previsión Social [STPS] puso en marcha un Programa de Autogestión en Seguridad y Salud en el Trabajo [PASST] en 2012, como respuesta al incremento de la tasa de accidentes en los centros de trabajo de los últimos años; asimismo, el Plan de Desarrollo 2007-2012 dio a conocer diversos objetivos para combatir esta problemática, de los cuales se pueden destacar el "Promover las políticas de Estado y generar las condiciones en el mercado laboral que incentiven la creación de empleos de alta calidad en el sector formal”, "Promover y Vigilar el Cumplimiento de la Normatividad Laboral", y "Elaborar e Instrumentar Acciones para Fortalecer la Seguridad y Salud en el Trabajo”, todo esto a través de una estructura y un esquema basados en los fundamentos legales de México (Secretaría del Trabajo y Previsión Social, 2015, pp. 5-23).

\section{Métodos de evaluación de riesgos}

El método Fine, fue publicado por William T. Fine en 1971 como un método de evaluación matemática para el control de riesgos, y está basada en tres factores: exposición o frecuencia con la que se produce la situación de riesgo o los sucesos que puedan iniciarlo; la probabilidad de que una vez que se haya dado la situación de riesgo, llegue a ocurrir el accidente; y las consecuencias, que representan los daños esperados o el grado de severidad que pueda causar el accidente; la multiplicación de estos factores da como resultado la magnitud del riesgo (Rubio \& Rubio, 2005, pp. 46-48). 


\section{El manual del sistema}

Mediante un proceso de mejora continua, y aplicando las leyes vigentes década país en materia de seguridad y salud de los empleados, los responsables deben crear una política de prevención de riesgos laborales, y lograr su cumplimiento a través de objetivos medibles en sus empresas; asimismo, debe incluir las metas y expectativas de la organización en cuanto al cuidado de la integridad física del empleado, las cuales van desde el compromiso de la alta dirección, hasta la responsabilidad de cada nivel jerárquico, así como asegurar que se dará seguimiento al sistema y se vigilara su cumplimiento a través de una evaluación continua de riesgos, que debe ser actualizada cada vez que se modifiquen las condiciones de trabajo; finalmente, mantener el control de la documentación y el registro de todos las actividades y acontecimientos en materia de prevención, tales como reglamentos, leyes, disposiciones, entre otras (Azcuénaga, 2004, pp. 16-21).

\section{Matriz de reporte de riesgos}

Con la intensión de conocer a detalle los riesgos, y teniendo en cuenta la probabilidad de ocurrencia de la causa raíz y las posibles consecuencias en rendimiento y costos, el Departamento de Defensa (2016, pp. 11-17), muestra un formato estándar para la evaluación y notificación de los resultados de los programas de evaluación de riesgos que facilita la comprensión en todos los niveles de gestión; dicho formato se representa en forma de una matriz denominada "Matriz de Información de Riesgos", en la cual se representa el nivel de los riesgos identificados con color verde [nivel de riesgo bajo], amarillo [riesgo moderado], y rojo [riesgo alto]. La matriz de información de riesgos es mostrada en la siguiente figura:

Figura 4. Matriz de información de riesgos.

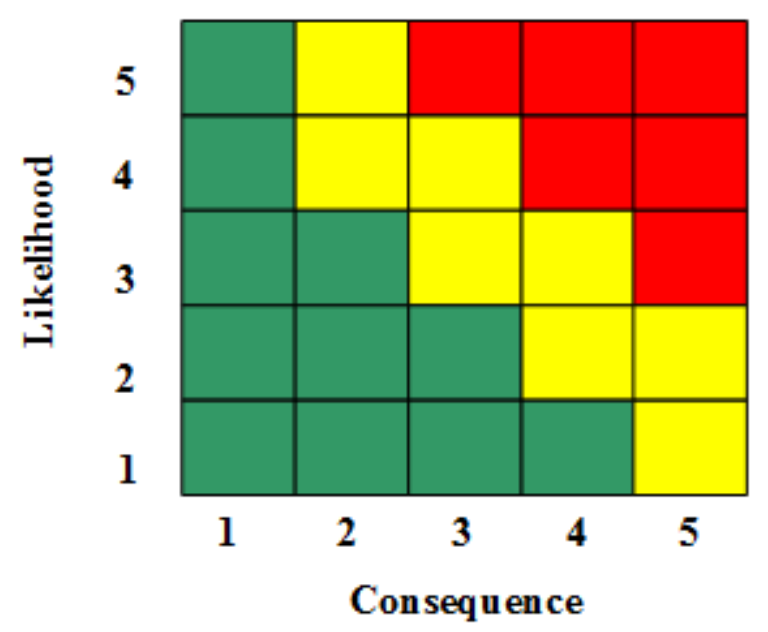

Fuente: Departamento de Defensa (2016, p. 11). 


\section{Riesgos de trabajo en el sistema de gestión de calidad}

Este artículo tiene como objetivo compartir una metodología para incorporar los riesgos de salud y seguridad al sistema de gestión de calidad certificado bajo la norma ISO 9001:2008, en una planta de elaboración de productos cárnicos, lo cual, es logrado mediante un método de investigación y acción planificado por etapas, iniciando por establecer un contexto por medio de la recopilación de información del sistema de gestión de calidad ISO y la normatividad de la OSHA; después, se realiza una tormenta de ideas para la identificación de peligros y riesgos de los procesos, para luego ser evaluados y valorados mediante un criterio obtenido de varios autores especializados en el tema; una vez realizado esto, se procede a implementar y mantener controles por medio de inspecciones, manuales o procedimientos de operación para equipos de alto riesgo y verificación de la funcionamiento de los controles; finalmente, la integración de los riesgos de seguridad y salud al sistema de gestión de calidad (Ulloa, 2012, pp. 101-108).

\section{La convergencia de lo cognitivo y lo social en los errores humanos}

Este artículo escrito por Anna Estany (2008, pp. 10-17), explica el factor del error humano en los accidentes laborales como consecuencia de la relación entre los aspectos cognitivos [errores] y sociales [tecnológicos], en el cual, el error se presenta como una secuencia planificada de actividades mentales o físicas que no consigue los fines deseados, y que son provocados principalmente por los sistemas tecnológicos y por el azar; asimismo, existen varios tipos de error, en los que se pueden destacar los errores activos y los latentes, los primeros están asociados con la actividad directa de los operarios en un sistema, mientras que los segundos son cometidos por los individuos alejados del control directo [por ejemplo, los diseñadores, personal de mantenimiento, dirigentes, entre otros], y que por lo general suelen tener consecuencias más peligrosas que los que están en "primera línea”.

\section{Barreras de protección}

Las barreras de protección consisten en la agrupación de equipamiento y/o controles administrativos que funcionan en conjunto con otras medidas de protección para mitigar o controlar los riesgos en los procesos, en otras palabras, este concepto identifica todos los "cinturones y tirantes" que intervienen en la protección al empleado frente a un evento peligroso o en la reducción de sus consecuencias (MacDonald, 2004, p. 49).

La Figura 5, muestra un modelo de reducción de riesgos utilizando el concepto de barreras de protección, el cual es muy eficaz para mostrar los detalles de su aplicación. 
Figura 5. Modelo de reducción de riesgos.

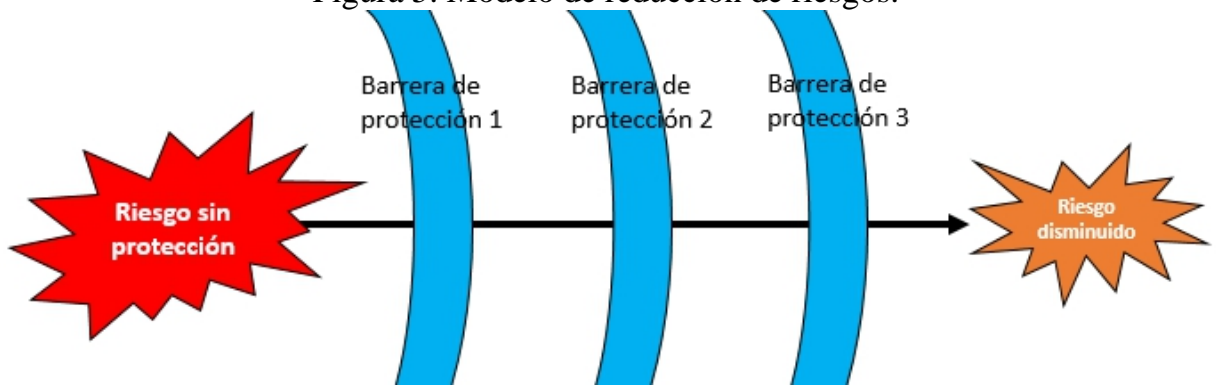

Fuente: Elaboración propia con base en MacDonald (2004, p. 56)

\section{Construyendo una cultura de seguridad}

Para Dekker (2014, p. 161), existen dos formas de ver la cultura de la seguridad: el punto de vista interpretativo, y el punto de vista funcionalista; sin embargo, no es de extrañarse que se culpe a la falta de "cultura de la seguridad" por los accidentes que ocurran, pero esto en realidad no ofrece una solución para reducir el problema; es por eso que, para influenciar en la cultura organizacional es necesario tener un nuevo punto de vista sobre la seguridad en las empresas, ya que en el "viejo" se toman a los errores humanos como la causa raíz de los problemas, y señalando a las personas como las causas de los problemas de seguridad, por lo cual, necesitan ser controlados, y por consecuencia, cambiar las actitudes y los comportamientos de los trabajadores eran el objetivo principal de los encargados. En la Tabla 1 se muestran algunas diferencias de la vieja forma de ver la seguridad industrial, con una más actualizada.

Tabla 1. Contraste de la vieja forma de ver la seguridad industrial con una más actualizada.

Viejo punto de vista

Las personas son vistas como un problema a controlar.

Enfoque en las actitudes y comportamientos de los trabajadores.

La seguridad es vista como la ausencia de eventos negativos (accidentes/incidentes).

Guiado por reglas y cumplimientos. Fuente: Elaboración propia con base en Dekker (2014, p. 163).

\section{La jerarquía de controles: Entendiendo la severidad y la reducción de riesgos}

Muchos de los profesionales de la seguridad y salud de los empleados, administran los riesgos únicamente con cálculos basados en ecuaciones de probabilidad, severidad y consecuencia, pero esto puede representar una sobrevaloración de los controles implementados para gestionar un riesgo específico, por lo que los administradores deben entender los efectos resultantes de una sobreestimación de los riesgos, así como su 
comprensión precisa, para poder proteger adecuadamente al trabajador (Burk, 2016, p. 37).

La American National Standard-Safety Machinery, presenta en 2005 una tabla de jerarquía de controles, en la cual, se muestra cómo cada nivel de control afecta a la severidad, así como las medidas de la reducción de riesgos, en donde el rediseño es considerado como la mejor clasificación para eliminar o sustituir los riesgos, y el equipo de protección personal la última opción. En la Tabla 2, se muestra la tabla con la jerarquía de control de riesgos completa.

Tabla 2. Jerarquía de control de riesgos.

\begin{tabular}{|c|c|c|c|}
\hline $\begin{array}{l}\text { Medidas de } \\
\text { reducción de } \\
\text { riesgos }\end{array}$ & Ejemplos & $\begin{array}{c}\text { Impacto en los factores } \\
\text { de riesgo }\end{array}$ & Clasificación \\
\hline $\begin{array}{l}\text { Eliminación o } \\
\text { Sustitución }\end{array}$ & $\begin{array}{c}\text { > Eliminar puntos críticos } \\
>\text { Procesos robotizados } \\
\text { > Rediseño de procesos }\end{array}$ & $\begin{array}{l}\text { Impacto en la severidad } \\
\text { y probabilidad del riesgo }\end{array}$ & Diseño \\
\hline $\begin{array}{l}\text { Guardas de } \\
\text { seguridad y } \\
\text { medidas } \\
\text { complementarias }\end{array}$ & $\begin{array}{c}\text { > Barreras } \\
>\text { Dispositivos de bloqueo } \\
>\text { Sensores de doble acción }\end{array}$ & $\begin{array}{l}\text { Gran impacto en la } \\
\text { probabilidad de daño. } \\
\text { Mínimo impacto en la } \\
\text { severidad en caso de } \\
\text { daño. }\end{array}$ & $\begin{array}{l}\text { Controles de } \\
\text { ingeniería }\end{array}$ \\
\hline $\begin{array}{l}\text { Dispositivos de } \\
\text { precaución }\end{array}$ & $\begin{array}{c}>\text { Alertas computarizadas } \\
>\text { Etiquetas y señalamientos } \\
\text { > Señales luminosas }\end{array}$ & \multirow{3}{*}{$\begin{array}{l}\text { Impacto potencial en la } \\
\text { probabilidad. No hay } \\
\text { impacto en la severidad. }\end{array}$} & \multirow{3}{*}{$\begin{array}{c}\text { Controles } \\
\text { administrativos }\end{array}$} \\
\hline $\begin{array}{l}\text { Entrenamiento y } \\
\text { Procedimientos }\end{array}$ & $\begin{array}{c}\text { > Procedimientos de trabajo } \\
\text { seguro } \\
>\text { Inspecciones de seguridad } \\
\text { de equipo } \\
>\text { Entrenamiento }\end{array}$ & & \\
\hline $\begin{array}{c}\text { Equipo de } \\
\text { Protección Personal }\end{array}$ & $\begin{array}{c}>\text { Guantes } \\
>\text { Lentes de seguridad } \\
>\text { Calzado de seguridad }\end{array}$ & & \\
\hline
\end{tabular}

Fuente: Elaboración propia con base en Burk (2016, p. 37).

\section{Evaluación de riesgos y jerarquía de control}

Para los responsables de la Seguridad y Salud de los empleados, la jerarquía de controles establece las acciones que deben ser considerados en un orden de eficacia para resolver situaciones de riesgo inaceptables, tomando en cuenta que se debe tener una secuencia en un orden descendente, en donde los intentos para eliminar o reducir los peligros son las medidas más efectivas, y se encuentran en la parte superior de la jerarquía; mientras que los pasos inferiores no son elegibles hasta agotar los superiores anteriores (Manuele, 2005, p. 36).

Normativas y directrices como la ANSI/ASSE Z244.1-2003, que aprobó el control de riesgos de energía; la ANSI B11.TR3-2000, ofrece una 
guía para estimar, evaluar y reducir los riesgos asociados con herramientas de trabajo; SEMI S2-0200, es un estándar internacional que ofrece una guía de equipo de manufactura de semiconductores; así como los ISO 12100-1 e ISO 14121/EN1050 para la seguridad en maquinaria y equipo, entre otras normas, tienen como requisito una evaluación de riesgos y el uso de una jerarquía de controles prescritos para la protección al trabajador (Manuele, 2005, pp. 33-34).

\section{Discusión y análisis futuro}

La administración de los riesgos laborales no se limita solamente a prevenir accidentes, se debe profundizar y analizar todos los factores que se involucran en un evento que daña la integridad de los trabajadores, como los factores psicológicos, sociales, y del medio ambiente. Tener una buena administración de riesgos no es solo emplear una herramienta que permita monitorear los riesgos presentes en las operaciones de una empresa, sino un conjunto de técnicas que permitan analizar de un modo más profundo el “comportamiento" de los riesgos, así como la metodología que se debe emplear para poder reducirlos o eliminarlos, técnicas que van desde barreras físicas de protección empleadas de manera ingenieril, hasta los estudios de comportamiento humano para evitar consecuencias potencialmente dañinas de los errores que las personas involuntariamente cometen.

Autores como Cuello, Pallares y Wehdeking (2008, p. 96), mencionan que, de un tiempo a la fecha, las empresas están expuestas a riesgos que pueden afectar el cumplimiento de su misión y sus objetivos institucionales; la administración de riesgos proporciona a las empresas los medios necesarios para identificarlos, para poder así medir su magnitud y determinar la forma de responder ante ellos, de manera que no se afecten los intereses, el patrimonio y la responsabilidad de la organización.

Para Abril, Enríquez y Sánchez (2006, p. 17), las empresas, buscan sistemas de gestión que se puedan implantar de manera sencilla, y a su vez, administrarlos de forma eficaz acorde a sus diferentes actividades para lograr permanecer en un entorno cada vez más competitivo; una clave para gestionar eficazmente la calidad y la prevención de riesgos laborales, es saber cómo satisfacer los requisitos reglamentarios y las demandas cada vez más exigentes de sus principales destinatarios: los clientes, la sociedad y los trabajadores, y que al mismo tiempo les permita ser competitivas.

A la vista de los resultados obtenidos de esta revisión de la literatura, se justifica un análisis de campo en el sector de la industria manufacturera de la ciudad, con el fin de evaluar el impacto de contar con una buena administración de riesgos laborales, en la competitividad de las empresas, esto puede ser logrado mediante la aplicación de encuestas a una muestra determinada; asimismo, se realizará un análisis con la información 
recolectada, para posteriormente desarrollar un proceso de administración de riesgos laborales que refleje un impacto o utilidad en la competitividad; asimismo, resulta viable realizar este estudio en Ciudad Juárez, ya que según el Instituto Nacional de Estadística y Geografía [INEGI], el 6.33\% del total nacional de la industria manufacturera se encuentra en esta ciudad, esto indica su importancia en el rubro o sector maquilador en la región.

\section{References:}

Abril, C., Enríquez, A., \& Sánchez, J. (2006). Manual para la integración de sistemas de gestión: Calidad, medio ambiente y prevención de riesgos laborales. Madrid: Fundación Confemetal.

Azcuénaga, L. (2004). El manual del sistema. En L. Azcuénaga, Guía para la implantación de un sistema de prevención de riesgos laborales (págs. 1523). Madrid: FC Editorial.

Burk, J. (2016). The Hierarchy of Controls: Understanding Severity \& Risk Reduction . Professional Safety, 37.

Cavassa, C. R. (1986). Seguridad industrial: Un enfoque integral. México: Ediciones Limusa.

Conesa, F. J., García, M. S., \& Lamata, M. T. (2012). Del dominó al queso suizo. La evolución en el campo de la seguridad laboral . Organización y dirección de empresas, 98-106.

Cortés, J. M. (2007). Técnicas de prevención de riesgos laborales; seguridad e higiene del trabajo. Madrid: Editorial Tébar.

Cuello, R., Pallares, L., \& Wehdeking, E. (2008). Aplicación del Estándar Australiano de Administración del Riesgo AS/NZS 4360:1999 en la empresa GECELCA. Pensamiento y Gestión, 94-112.

Dekker, S. (2014). Building a Safety Culture. En S. Dekker, The Field Guide to Understanding 'Human Error' (págs. 161-190). Surrey, England: Ashgate.

Department of Defense. (10 de Febrero de 2016). Defense Acquisition University. Obtenido de Office of the Under Secretary of Defense for Acquisition, Technology and Logistics: http://www.acq.osd.mil/se/docs/DoD-Risk-Mgt-Guide-v7-interimDec2014.pdf

Estany, A. (2008). La convergencia de lo cognitivo y lo social en los errores humanos. Tópicos, 9-35.

Gallegos, W. L. (2012). Revisión histórica de la salud ocupacional y la seguridad industrial. Revista Cubana de Salud y Trabajo, 45-52.

Garachana, H. L. (1999). Seguridad industral y protección ambiental para la pequeña y mediana empresa. México: Universidad Iberoamericana.

Gawande, A. (2010). The Checklist Manifesto: How to Get Things Right. New York: Metropolitan Books. 
MacDonald, D. (2004). Hazards and risk reduction. En D. MacDonald, Industrial Safety, Risk Assessment and Shutdown Systems (págs. 33-64). Burlington: Newnes.

Manuele, F. (2005). Risk Assessment \& Hierarchies of Control. Professional Safety, 33-39.

Menéndez, F. (2007). Técnicas de prevención de riesgos laborales. En F. Menéndez, Formación superior en prevención de riesgos laborales: parte obligatoria y común (págs. 75-83). Valladolid: Lex Nova.

Muschara, T. (2010). General Electric Error Prevention Skills for FrontLine Personnel. Marietta, Georgia: Muschara Management Consulting.

Professional Safety. (2007). Industry Notes. Professional Safety, 26-31.

Radvanska, A. (2010). Accident losses elimination by means of safety pyramid analysis. International Journal of Engineering, 73-76.

Rubio, J. C., \& Rubio, M. (2005). Manual para la formación de nivel superior en prevención de riesgos laborales. España: Ediciones Díaz De Santos.

Saenz-Calcedo, J. G., González, A. G., \& Et al. (2015). Analysis on integrated management of the quality, environment and safety on the industrial projects . Elsevier, 140-145.

Secretaría del Trabajo y Previsión Social. (9 de Septiembre de 2015). Secretaría del Trabajo y Previsión Social. Obtenido de Autogestión en Seguridad $y$ Salud en el Trabajo: http://autogestion.stps.gob.mx:8162/pdf/Lineamientos\%20Generales\%20200 8.pdf

Secretaría del Trabajo y Previsión Social. (9 de Septiembre de 2015). STPS. Obtenido de Secretaría del Trabajo y Previsión Social: http://www.stps.gob.mx/bp/secciones/conoce/quienes_somos/quienes_somos /historia_stps.htm

Ulloa, M. Á. (2012). Riesgos del Trabajo en el Sistema de Gestión de Calidad . Ingeniería Industrial, 100-111. 